\title{
Detection of early cartilage deterioration associated with meniscal tear using T1 $\rho$ mapping magnetic resonance imaging
}

\author{
Hirokazu Matsubara', Ken Okazaki i* Y Yukihisa Takayama², Kanji Osaki ${ }^{1}$, Yoshio Matsuo $^{2}$, Hiroshi Honda²
} and Yukihide Iwamoto ${ }^{1}$

\begin{abstract}
Background: In patients with degenerative meniscal tears, subclinical cartilage degeneration may be present even if gross morphological changes are not evident. The aim of this study was to detect occult cartilage degeneration using T1p MRI mapping in patients with meniscal tears without obvious radiographic osteoarthritis (OA).

Methods: A total of 22 subjects with degenerative meniscal tears in the early stages of osteoarthritis [Kellgren-Lawrence $(\mathrm{KL})$ grade of 0-2] and 19 healthy subjects as the control group were examined. The femoral condyle was divided into four $30^{\circ}$ wedges $\left(-30^{\circ}-0^{\circ}\right.$ anteriorly, $0^{\circ}-30^{\circ}, 30^{\circ}-60^{\circ}$ and $60^{\circ}-90^{\circ}$ posteriorly), and each area of cartilage was further divided into superficial and deep layers of equal thickness. The tibial side was divided into anterior and posterior areas with superficial and deep layers in each. The mean T1 $\rho$ values (ms) in each area were calculated.

Results: On the femoral side, $T 1 \rho$ values of the superficial and deep regions $\left(-30^{\circ}-0^{\circ}, 0^{\circ}-30^{\circ}\right.$ and $\left.30^{\circ}-60^{\circ}\right)$ in the meniscal tear group were significantly higher than those in the control group [superficial $\left(-30^{\circ}-0^{\circ}\right): 49.0 \pm 4.0$ (meniscal tear group) vs $45.1 \pm 2.1$ (control group), deep $\left(-30^{\circ}-0^{\circ}\right): 45.2 \pm 3.3$ vs $39.5 \pm 5.0$, superficial $\left(0^{\circ}-30^{\circ}\right): 54.5 \pm 5.3$ vs $47.4 \pm$ 5.7, deep $\left(0^{\circ}-30^{\circ}\right): 46.8 \pm 4.0$ vs $40.7 \pm 6.3$, superficial $\left(30^{\circ}-60^{\circ}\right): 50.5 \pm 3.1$ vs $\left.47.1 \pm 5.7\right]$. On the tibial side, the meniscal tear group had significantly higher T1 $\rho$ values superficially in both anterior and posterior regions compared with the control group [superficial (anterior): $52.0 \pm 4.3$ vs $46.7 \pm 5.4$, superficial (posterior): $53.1 \pm 5.1$ vs $46.0 \pm 4.9$ ]. Moreover, these significant differences were observed when comparing patients in the meniscal tear group with $\mathrm{KL}$ grades of 0 or 1 and the control group.

Conclusions: Our study suggested that early biochemical changes in cartilage associated with degenerative meniscal tears occur first in the superficial zones in areas of contact during slight flexion. Characterising the early relationship between cartilage degeneration and degenerative meniscal tears using T1 $\rho$ MRI mapping may be of clinical benefit and provide further evidence linking meniscal injury to OA.
\end{abstract}

Keywords: Cartilage degeneration, Meniscus, Osteoarthritis, Magnet resonance imaging (MRI), T1p MRI

\section{Background}

Despite the high prevalence of meniscal tears, the role of meniscal injury in the pathogenesis of articular cartilage degeneration is poorly understood [1]. The menisci play an important role in knee joint stability, joint lubrication and shock absorption and help to maintain the integrity of the articular cartilage [2,3]. A number of studies have

\footnotetext{
* Correspondence: okazaki@med.kyushu-u.ac.jp

${ }^{1}$ Department of Orthopedic Surgery, Graduate School of Medical Sciences, Kyushu University, 3-1-1 Maidashi, Higashi-ku, Fukuoka 812-0054, Japan Full list of author information is available at the end of the article
}

shown an association between knee osteoarthritis (OA) and meniscal damage [4-8]. These findings support the theory that disruption and excision of the meniscus contribute to cartilage degeneration or progression of OA. We have encountered several cases in which hydrarthrosis with subsequent progressive cartilage degeneration occurred after meniscectomy, although obvious morphological changes had not been detected during preoperative evaluation using radiography and conventional magnetic resonance imaging (MRI) or during intraoperative arthroscopy. We hypothesise that subclinical 
cartilage degeneration occurs after the occurrence of a degenerative meniscal tear, even if gross morphological changes are not evident in the cartilage. Meniscectomy is commonly performed on a patient with knee joint symptoms despite being associated with a mild OA such as that of Kellgren and Lawrence grade 2. Therefore, it would be a clinical benefit if the surgeon could preoperatively evaluate occult cartilage degeneration in patients with a meniscal tear. In addition, characterising the early relationship between cartilage degeneration and meniscal damage may provide further evidence linking meniscal injury to OA.

Early stages of OA are primarily associated with loss of proteoglycans (PG), changes in the water content and minor structural changes in collagen [9]. MRI techniques have the potential to detect such biochemical changes in the composition of joint articular cartilage non-invasively $[10,11]$. Specifically, T1 $\rho$ mapping has been shown to be sensitive to changes in PG loss in cartilage and has attracted attention as a non-invasive and a quantitative means of detecting biochemical changes in cartilage degeneration prior to morphological or clinical changes [10-12]. Previous studies have demonstrated that T1 $\rho$ values are elevated in the cartilages of OA patients when compared with corresponding healthy subjects $[13,14]$. T1 $\rho$ values are correlated with the severity of macroscopic cartilage damage and histologically-assessed PG content in the cartilages of patients with OA or rheumatoid arthritis [15]. Therefore, we used T1 $\rho$ mapping to detect early cartilage degeneration associated with degenerative meniscal tears.

The purpose of our study was (1) to detect occult cartilage degeneration in patients with degenerative meniscal tears using T1 $\rho$ MRI mapping, (2) to investigate the distribution of $\mathrm{T} 1 \rho$ values in the superficial and deep layers of adjacent femoral and tibial cartilages and (3) to characterise the relationship between early cartilage degeneration and degenerative meniscal tears.

\section{Methods}

\section{Subjects}

This study was granted Institutional Review Board approval at Kyushu University and complied with the Ethical Committee Standards (approval number: 23-75). All subjects provided written informed consent prior to the study. The subjects were recruited from the patients who visited our hospital due to knee joint symptoms. Table 1 shows the baseline characteristics of the subjects included in the study. Anthropometric measurements, including height and weight, were collected, and body mass index (BMI) was calculated for all participants. MRI and radiographic examination was performed on all subjects. Meniscal lesions were graded based on MRI images using the following grading system: grade 0 ,
Table 1 Baseline characteristics and radiographic parameters of meniscal tear group and control group

\begin{tabular}{llll}
\hline & $\begin{array}{l}\text { Meniscal tear } \\
\text { group }\end{array}$ & $\begin{array}{l}\text { Control } \\
\text { group }\end{array}$ & P value \\
\hline Characteristic & 22 & 19 & \\
Participants, no & $57.0 \pm 14.1$ & $39.0 \pm 7.2$ & $<0.05$ \\
Age, mean $\pm \mathrm{SD}$, years & $24.4 \pm 2.6$ & $23.9 \pm 2.1$ & 0.31 \\
BMI, mean $\pm \mathrm{SD}, \mathrm{kg} / \mathrm{m}^{2}$ & & & \\
Radiographic parameter & & $176.7 \pm 0.8$ & 0.64 \\
FTA, mean $\pm \mathrm{SD},{ }^{\circ}$ & $177.0 \pm 2.6$ & & \\
KL grade, no & & 16 & \\
Grade 0 & 4 & 3 & \\
Grade1 & 8 & 0 & \\
Grade2 & 10 & & \\
\hline
\end{tabular}

BMI body mass index, FTA femoro-tibial angle, KL Kellgren and Lawrence.

normal meniscus; grade 1, increased signal intensity of the meniscus without evidence of a tear; grade 2, small radial meniscal tear; grade 3 , non-displaced single meniscal tear; grade 4, non-displaced complex meniscal tear; grade 5, meniscal tear with displaced component; and grade 6, macerated meniscal tear [16]. Patients with meniscal changes scoring 2-6 on MRI were assigned to the meniscal tear group. Femoro-tibial angle (FTA) values were calculated based on radiographs, and radiographic severity of OA was determined according to the Kellgren and Lawrence (KL) grading system. The meniscal tear group comprised 22 subjects: Fifteen males and seven females ranging in age from 32 to 73 years (mean, $56.4 \pm 12.8$ years) with KL grades between 0 and 2. Only patients with frequent symptoms, defined as pain in or around the knee on most days for at least 1 month over the past 12 months and a positive McMurray test, were investigated. Subjects who had severe OA or KL grades more than 3 were excluded, as were subjects with a ligament injury and/or cartilage injury on MRI. To categorise the severity of OA, subjects in the meniscal tear group were further divided into two subgroups: 'KL normal' with a KL grade of 0 or $1(\mathrm{n}=12)$ and 'KL mild OA' with a KL grade of $2(n=10)$. The meniscal tear group consisted of 20 medial and two lateral meniscal tears. The control group comprised 19 healthy individuals, all males and ranging in age from 28 to 54 years (mean, $39.0 \pm 7.2$ years) without any clinical symptoms of OA or other knee injuries. All controls were only enrolled in the study if they had a KL grade of 0 , indicating no signs of radiographic $\mathrm{OA}$.

\section{Magnetic resonance imaging protocols}

MRI was performed on a 3-Tesla system (Achieva 3.0 T, Quasar Dual, Philips Healthcare, Best, the Netherlands) using an 8-channel phased-array knee coil. Sagittal fat-suppression turbo spin echo T2-weighted images 
(FS-T2WI) were obtained using the following parameters: Repetition time/echo time $(\mathrm{TR} / \mathrm{TE})=4,675 / 71 \mathrm{~ms}$, field of view $(\mathrm{FOV})=140 \times 140 \mathrm{~mm}$, matrix $=400 \times 400$, slice thickness $=3 \mathrm{~mm}$, slice gap $=0 \mathrm{~mm}$, flip angle $=$ $90^{\circ}$, bandwidth $=31.54 \mathrm{~Hz} /$ pixel, number of slices $=26$ and total scan time $=3 \mathrm{~min} 33 \mathrm{~s}$. FS-T2WI was used as an anatomical reference.

Two-dimensional (2D)-Sagittal T1 $\rho$ mapping was calculated from T1 $\rho$-prepared images using the fast field echo technique. The imaging parameters were as follows: $\mathrm{TR} / \mathrm{T}=4.7 / 2.4 \mathrm{~ms}, \mathrm{FOV}=140 \times 140 \mathrm{~mm}$, matrix $=320 \times 320$, slice thickness $=3 \mathrm{~mm}$, slice gap $=$ $0 \mathrm{~mm}$, flip angle $=35^{\circ}$, bandwidth $=31.54 \mathrm{~Hz} /$ pixel, spin-lock pulses $=20 / 1 / 40 / 60 / 80 \mathrm{~ms}$, spin-lock pulse frequency $=500 \mathrm{~Hz}$, number of slices $=26$ and total scan time $=16 \min 15 \mathrm{~s}$. We used a low flip angle, but it did not affect T1 $\rho$ contrast, since we used 6,000 ms of shot intervals between each slice acquisition and filled the k-space using low-high ordering. T1p mapping was produced with Philips Research Integrated Development Environment (PRIDE) software written in
Interactive Data Language (IDL 6.3, ITT Inc. Boulder, CO, USA) and was used in the quantitative assessment.

\section{Imaging assessment of T1 $\rho$ maps}

Mean T1 $\rho$ values and standard deviations were calculated by two orthopaedic surgeons (H.M. and K.O., with nine and eight years' experience, respectively) using the 'Medical Image Processing, Analysis, and Visualization' software (MIPAV, Biomedical Imaging Research Services Section, Center for Information Technology, National Institutes of Health, Bethesda, MD, USA) (Figure 1a). On the femoral side, regions of interest (ROIs) in the articular cartilage were divided into anterior and posterior parts. The posterior part was further divided into three $30^{\circ}$ wedges since the medial femoro-tibial contact point shifts posteriorly until $60^{\circ}$ of flexion $[17,18]$, which increases the risk of meniscal injury more than in extended positions. Furthermore, we distinguished superficial and deep layers of equal thickness in all areas (Figure 1b). ROIs of the articular cartilage on the tibial side were divided into anterior and posterior areas, and

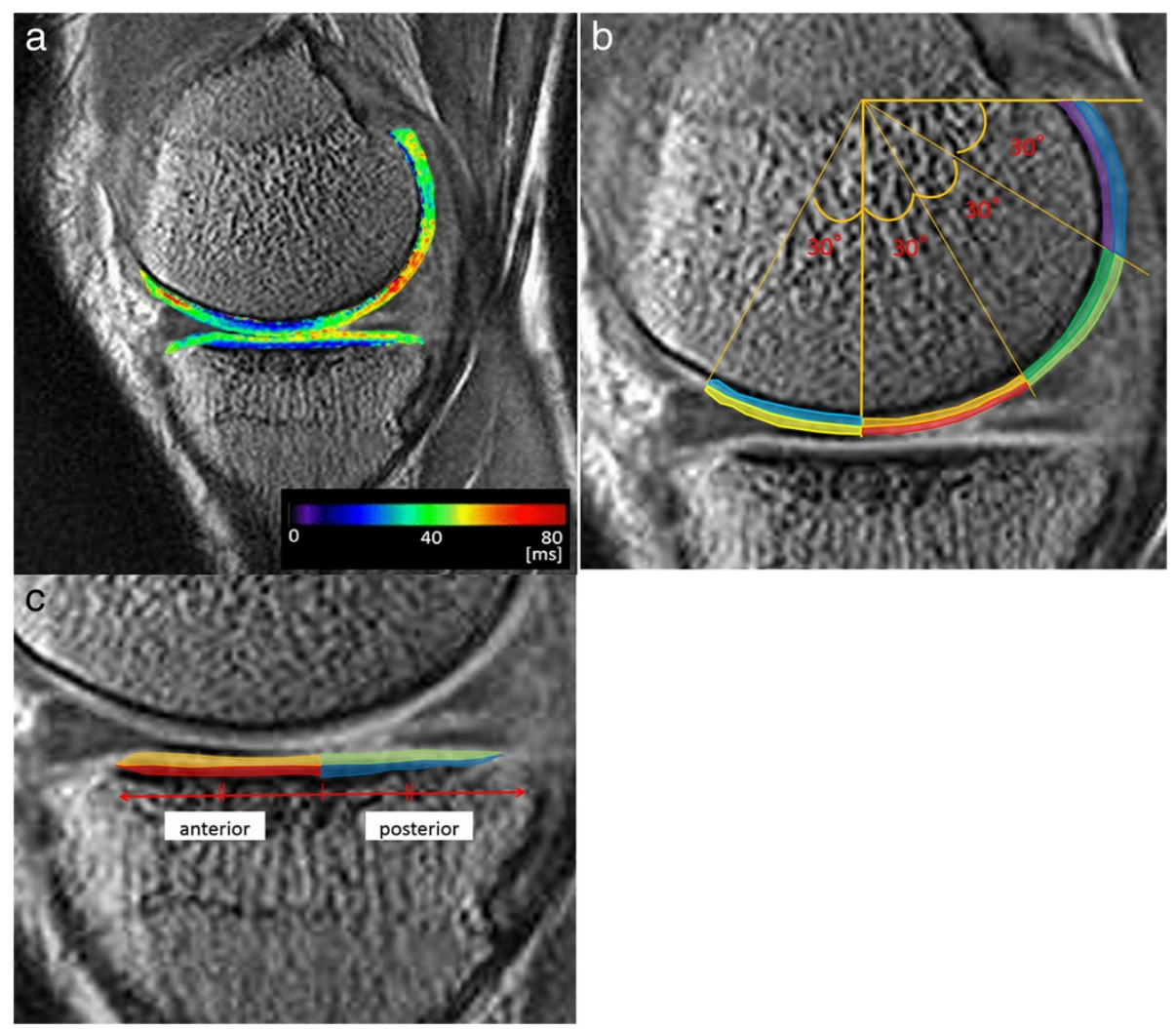

Figure 1 Assessment of T1 $\rho$ values in the articular cartilage. a. Colored T1 $\rho$ mappings of femoral and tibial articular cartilage. b. Regions of interest (ROls) were divided into eight regions on the femoral articular cartilage; superficial $\left(-30^{\circ}-0^{\circ}\right)$ : yellow; deep $\left(-30^{\circ}-0^{\circ}\right)$ : cyan; superficial $\left(0^{\circ}-30^{\circ}\right)$ : red; deep $\left(0^{\circ}-30^{\circ}\right)$ : orange; superficial $\left(30^{\circ}-60^{\circ}\right)$ : yellow-green; deep $\left(30^{\circ}-60^{\circ}\right)$ : green; superficial $\left(60^{\circ}-90^{\circ}\right)$ : blue; deep $\left(60^{\circ}-90^{\circ}\right)$ : purple. c. ROls were divided into four regions on the tibial cartilage; superficial (anterior): orange; deep (anterior): red; superficial (posterior): yellow-green; deep (posterior): blue. 
superficial and deep layers were analysed separately (Figure 1c). In addition, anterior and posterior horn of the meniscus on the same MRI slice was also analyzed. Three adjacent slices at the centre of the medial or lateral compartment in the sagittal plane, respectively, were analysed for each subject. After analysing the ROIs for each of the 14 areas (femoral side, eight areas; tibial side, four areas; meniscus, two areas) on each of the three slices, mean T1 $\rho$ values and standard deviations were used for the statistical analyses.

\section{Statistical analyses}

All data are expressed as the mean \pm SD. A MannWhitney $\mathrm{U}$ test was performed to compare $\mathrm{T} 1 \rho$ values for each area on the femoral and tibial articular cartilage between the meniscal tear group and the control group and between genders. Analysis of variance (ANOVA) and a post hoc comparison using Tukey's honest significant difference test were used to assess differences in $\mathrm{T} 1 \rho$ values for each area on the femoral and tibial articular cartilage between the control, the KL normal and KL mild OA groups. In addition, Pearson's correlation analysis was applied to study the relationship between patient age and $\mathrm{T} 1 \rho$ values for each area on the femoral and tibial articular cartilage of both the meniscal tear and the control groups. The intra-class correlation coefficient (ICC) was used to describe inter-observer agreement for the measurement of the T1 $\rho$ values. ICCs close to 1.0 indicated good agreement between the two observers. All statistical tests were performed with the JMP software version 9.0 (SAS Institute, Cary, NC, USA). A P-value $<0.05$ was considered statistically significant for each two-tailed analysis.

\section{Results}

T1 $\rho$ values in each area of the articular cartilage on the femoral condyle and tibial plateau are summarized in Table 2 and Table 3, respectively. For the femoral condyle, T1 $\rho$ values in the meniscal tear group were significantly higher than those in the control group in both the superficial and deep regions of the $-30^{\circ}-0^{\circ}$ wedge and the $0^{\circ}-30^{\circ}$ wedge, and in the superficial region of the $30^{\circ}-60^{\circ}$ wedge (Table 2 and Figure 2a). With regard to the differences in OA severity between the control group and the two subgroups, a statistically significant difference was observed in both the superficial and deep regions of the $-30^{\circ}-0^{\circ}$ wedge and the $0^{\circ}-30^{\circ}$ wedge between the control group and the $\mathrm{KL}$ normal group and between the control group and the KL mild OA group. However, no significant differences were observed between the two subgroups in the meniscal tear group (Table 2 and Figure 2b). No statistically significant differences were observed between the genders as well (Table 2 and Figure 2c).

For the tibial plateau, the meniscal tear group had significantly higher $\mathrm{T} 1 \rho$ values than the control group in both the anterior and posterior regions in the superficial areas (Table 3 and Figure 3a). With regard to the differences between the control group and the two subgroups in the meniscal tear group, a statistically significant difference was observed in the superficial areas of both the anterior and posterior regions between the control group and the KL normal group, and between the control group and the KL mild OA group. However, no statistically significant differences were observed between the two subgroups in the meniscal tear group or between the genders, similarly to the findings related to the femoral condyle (Table 3 and Figure $3 b$ and $c$ ). The $\mathrm{T} 1 \rho$ values (ms) of the posterior horn of meniscus were significantly higher in the meniscal tear group than those in the control group $(44.2 \pm 3.5$ vs $35.6 \pm 2.1$, respectively, $\mathrm{p}<0.01$ ). There were no significant differences in T1 $\rho$ values of the anterior horn of meniscus between in the meniscal tear group and the control group (36.0 \pm 2.1 vs $34.4 \pm 3.1$, respectively).

No significant correlations were observed between age and T1 $\rho$ values at any regions of both the femoral condyle and the tibial plateau in the meniscal tear group (Figure 4a and b), but slight or moderate correlations were observed between these in the control group (Figure $4 \mathrm{c}$ and $\mathrm{d}$ ). The intra-class correlation coefficient (ICCs) of the T1 $\rho$ values between the two observers was $0.93(95 \% \mathrm{CI}=0.84-0.95)$, indicating good agreement between the observers.

\section{Discussion}

In the present study, we evaluated sub-regional and layer-specific T1 $\rho$ values of femoro-tibial articular cartilage in patients with meniscal tears with no or mild radiographic $\mathrm{OA}$ and compared them with those of healthy subjects. Higher T1 $\rho$ values were observed at the distal area of the femoral condyle and the tibial plateau in the meniscal tear group, even in the subgroup with no radiographic OA. Hence, the study suggested the association between meniscal tears and occult cartilage damage.

However, this study had several limitations. First, the mean age was different between the meniscal tear group and the control group, and the average age of the subjects in the meniscal tear group was relatively high. It was challenging to recruit age-matched control subjects from volunteers. In fact, there were weak or moderate correlations between age and $\mathrm{T} 1 \rho$ values in articular cartilage in the control group. However, no correlations were observed between age and $\mathrm{T} 1 \rho$ values in the meniscal tear group. Patients less than 50 years old with 
Table 2 T1 $\rho$ values ( $m s$ ) for articular cartilage on the femoral condyle

\begin{tabular}{|c|c|c|c|c|c|c|c|c|}
\hline & Superficial $\left(-30^{\circ}-0^{\circ}\right)$ & Deep $\left(-30^{\circ}-0^{\circ}\right)$ & Superficial $\left(0^{\circ}-30^{\circ}\right)$ & Deep $\left(0^{\circ}-30^{\circ}\right)$ & Superficial $\left(30^{\circ}-60^{\circ}\right)$ & Deep $\left(30^{\circ}-60^{\circ}\right)$ & Superficial $\left(60^{\circ}-90^{\circ}\right)$ & Deep $\left(60^{\circ}-90^{\circ}\right)$ \\
\hline Meniscal tear group & $49.0 \pm 4.0$ & $45.2 \pm 3.3$ & $54.6 \pm 5.3$ & $46.8 \pm 4.0$ & $50.5 \pm 3.1$ & $52.2 \pm 3.6$ & $45.4 \pm 3.1$ & $46.2 \pm 3.4$ \\
\hline Control group & $45.1 \pm 2.1$ & $39.6 \pm 5.0$ & $47.4 \pm 5.7$ & $40.7 \pm 6.3$ & $47.1 \pm 5.7$ & $47.8 \pm 7.6$ & $42.5 \pm 5.8$ & $42.9 \pm 6.4$ \\
\hline P-value & $<0.05$ & $<0.05$ & $<0.05$ & $<0.05$ & $<0.05$ & 0.08 & 0.29 & 0.15 \\
\hline \multicolumn{9}{|c|}{ Comparison between the OA severity groups } \\
\hline Normal $(n=12)$ & $47.7 \pm 3.8$ & $47.1 \pm 4.0$ & $53.2 \pm 6.3$ & $46.1 \pm 4.2$ & $49.8 \pm 3.5$ & $51.9 \pm 3.5$ & $45.0 \pm 4.0$ & $46.3 \pm 4.2$ \\
\hline Mild OA $(n=10)$ & $48.2 \pm 3.3$ & $48.9 \pm 4.2$ & $56.0 \pm 4.4$ & $47.1 \pm 4.0$ & $51.1 \pm 3.5$ & $52.5 \pm 4.0$ & $45.0 \pm 2.3$ & $45.8 \pm 2.5$ \\
\hline P-value & 0.88 & 0.72 & 0.18 & 0.87 & 0.62 & 0.84 & 0.87 & 0.71 \\
\hline \multicolumn{9}{|c|}{ Comparison between genders } \\
\hline Male $(n=15)$ & $47.7 \pm 3.7$ & $45.3 \pm 3.5$ & $54.6 \pm 5.2$ & $46.9 \pm 4.1$ & $50.1 \pm 3.5$ & $52.6 \pm 4.2$ & $46.0 \pm 3.6$ & $46.3 \pm 4.1$ \\
\hline Female $(n=7)$ & $48.2 \pm 4.5$ & $46.7 \pm 4.1$ & $54.7 \pm 6.0$ & $46.8 \pm 4.0$ & $51.4 \pm 2.5$ & $51.5 \pm 2.4$ & $44.0 \pm 1.9$ & $46.2 \pm 2.0$ \\
\hline P-value & 0.25 & 0.32 & 0.33 & 0.80 & 0.29 & 0.53 & 0.32 & 1.00 \\
\hline
\end{tabular}


Table 3 T1p values (ms) for articular cartilage on the tibial plateau

\begin{tabular}{|c|c|c|c|c|}
\hline & Superficial (anterior) & Deep (anterior) & Superficial (posterior) & Deep (posterior) \\
\hline Meniscal tear group & $52.0 \pm 4.3$ & $41.3 \pm 3.7$ & $53.1 \pm 5.1$ & $42.8 \pm 4.3$ \\
\hline Control group & $46.7 \pm 5.4$ & $39.6 \pm 5.0$ & $46.0 \pm 4.9$ & $41.3 \pm 4.8$ \\
\hline P-value & $<0.05$ & 0.28 & $<0.05$ & 0.55 \\
\hline \multicolumn{5}{|c|}{ Comparison between the $\mathrm{OA}$ severity groups } \\
\hline Normal $(n=12)$ & $51.7 \pm 4.5$ & $41.6 \pm 3.9$ & $52.9 \pm 5.5$ & $43.0 \pm 4.9$ \\
\hline Mild OA $(n=10)$ & $51.8 \pm 4.3$ & $40.4 \pm 3.3$ & $52.3 \pm 4.8$ & $41.6 \pm 3.6$ \\
\hline P-value & 1.00 & 0.49 & 0.37 & 0.22 \\
\hline \multicolumn{5}{|c|}{ Comparison between genders } \\
\hline Male $(n=15)$ & $52.5 \pm 5.0$ & $41.6 \pm 4.3$ & $55.0 \pm 4.7$ & $42.5 \pm 4.7$ \\
\hline Female $(n=7)$ & $50.6 \pm 2.4$ & $40.6 \pm 2.3$ & $51.0 \pm 5.3$ & $42.4 \pm 4.1$ \\
\hline P-value & 0.70 & 0.64 & 0.19 & 0.97 \\
\hline
\end{tabular}

meniscal tears also showed relatively high $\mathrm{T} 1 \rho$ values. Therefore, the increased T1 $\rho$ values in the meniscal tear group were not attributed to the relatively higher age of this group. Second, the magic angle effect may affect the calculation of $\mathrm{T} 1 \rho$ values in articular cartilage, especially in the $30^{\circ}-60^{\circ}$ area. This area is susceptible to the magic angle effect both superficially and deeply since this effect increases T1 $\rho$ values relating to the orientation of collagen fibrils in articular cartilage and a static magnetic field $[19,20]$. Therefore, attention should be paid to results showing that $\mathrm{T} 1 \rho$ values are significantly higher in this area, when comparing different areas in the same
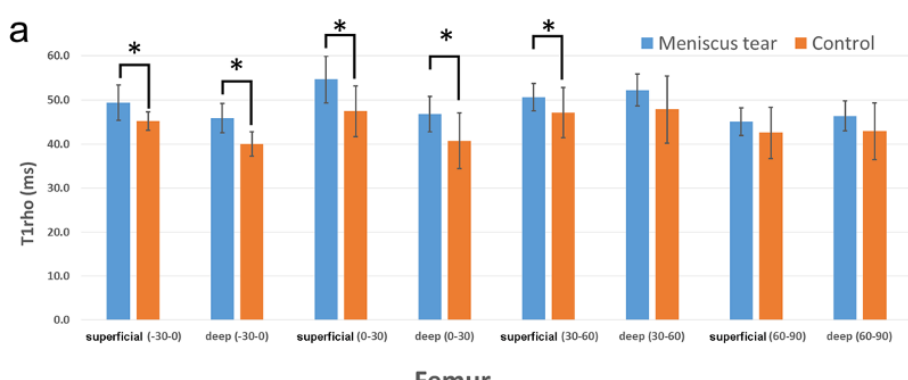

Femur
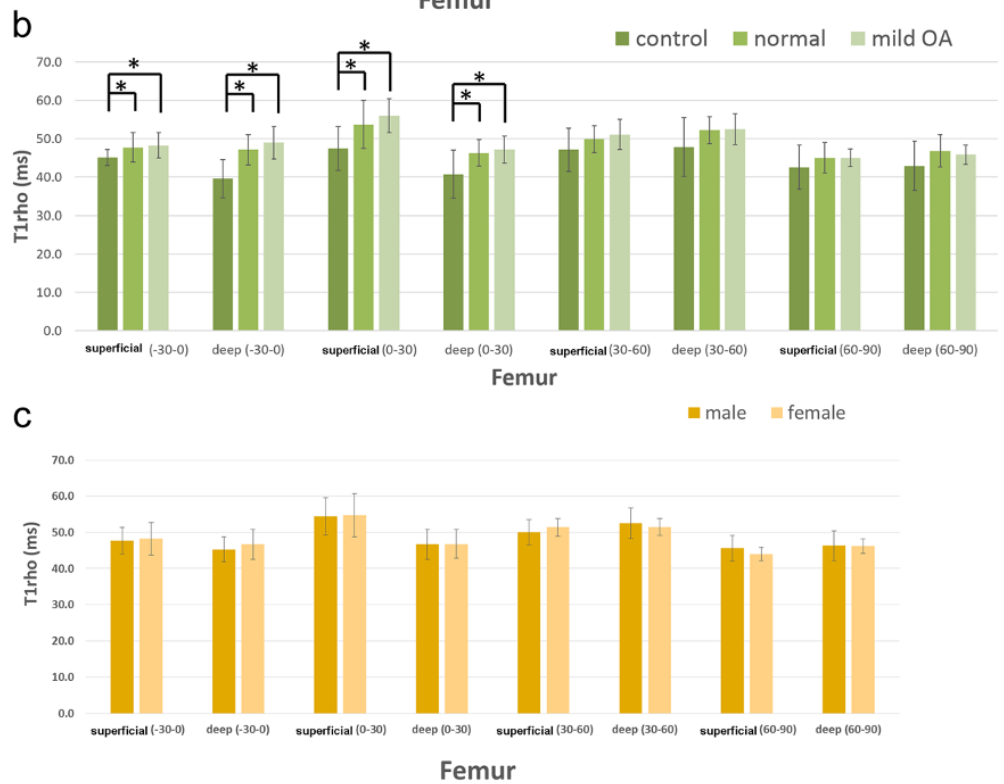

Figure 2 T1 $\rho$ values in different cartilage regions of the femoral condyle. $\mathbf{a}$. Meniscal tear and control groups $\mathbf{b}$. Normal and mild OA $\mathbf{c}$. Males and females. Error bars: 95\% confidence interval *: $P<0.05$. 


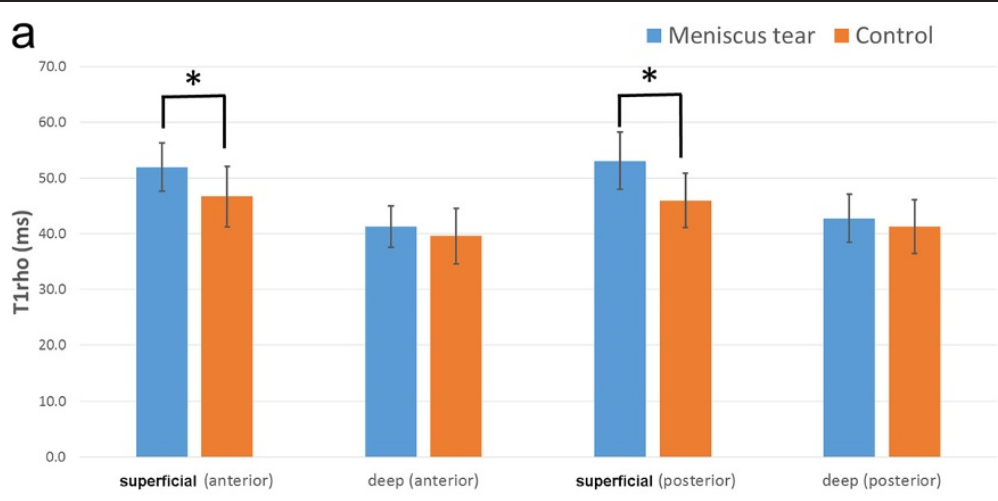

Tibia

b
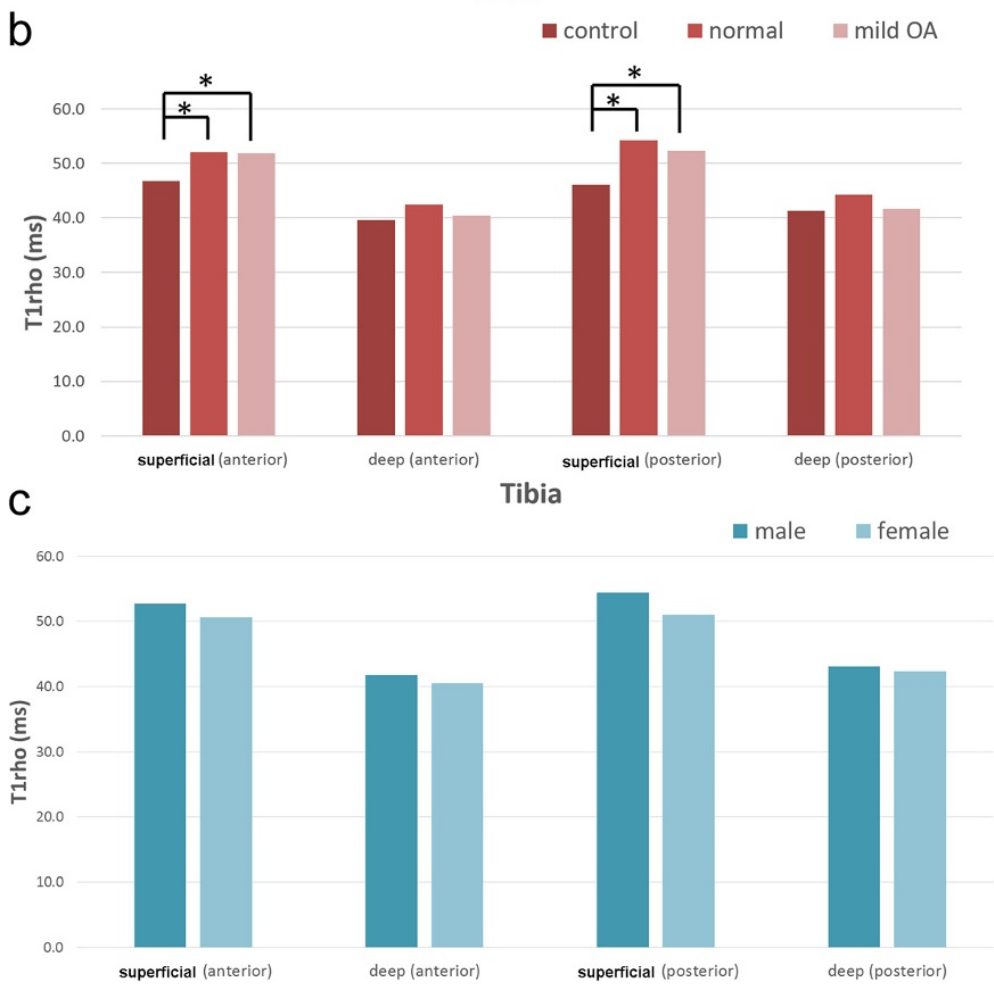

Figure 3 T1 $\rho$ values in different cartilage regions of the tibial plateau. a. Meniscal tear and control groups. b. Normal and mild OA. c. Males and females. Error bars: 95\% confidence interval *: $\mathrm{P}<0.05$.

knee. However, this study compared the same area between groups of subjects, indicating that $\mathrm{T} 1 \rho$ values in both superficial and deep regions of the $30^{\circ}-60^{\circ}$ area in the meniscal tear group were significantly higher than those of the control group. Therefore, there is no doubt that cartilage denaturation had occurred in association with a degenerative meniscal tear in these areas. Third, ROIs were manually drawn around the articular cartilage boundaries to calculate T1 $\rho$ values. Factors that might cause miscalculation include the misidentification of articular cartilage boundaries, such as that between the superficial and deep layers. However, ICCs indicated good agreement between the observers, and so any accidental error associated with drawing the ROIs would not significantly affect the conclusions of this study. Finally, the relatively small sample size and the crosssectional design are other limitations of the study. It was also difficult to evaluate other potential confounders influencing the $\mathrm{T} 1 \rho$ values with multivariable analysis.

Previous studies have evaluated the relationship between cartilage change and OA using a variety of methods. Bassiouni et al. examined the cartilage in OA using phonoarthrography, musculoskeletal ultrasonography and biochemical biomarkers and concluded that phonoarthrography and musculoskeletal ultrasonography could be used as parameters for following up 

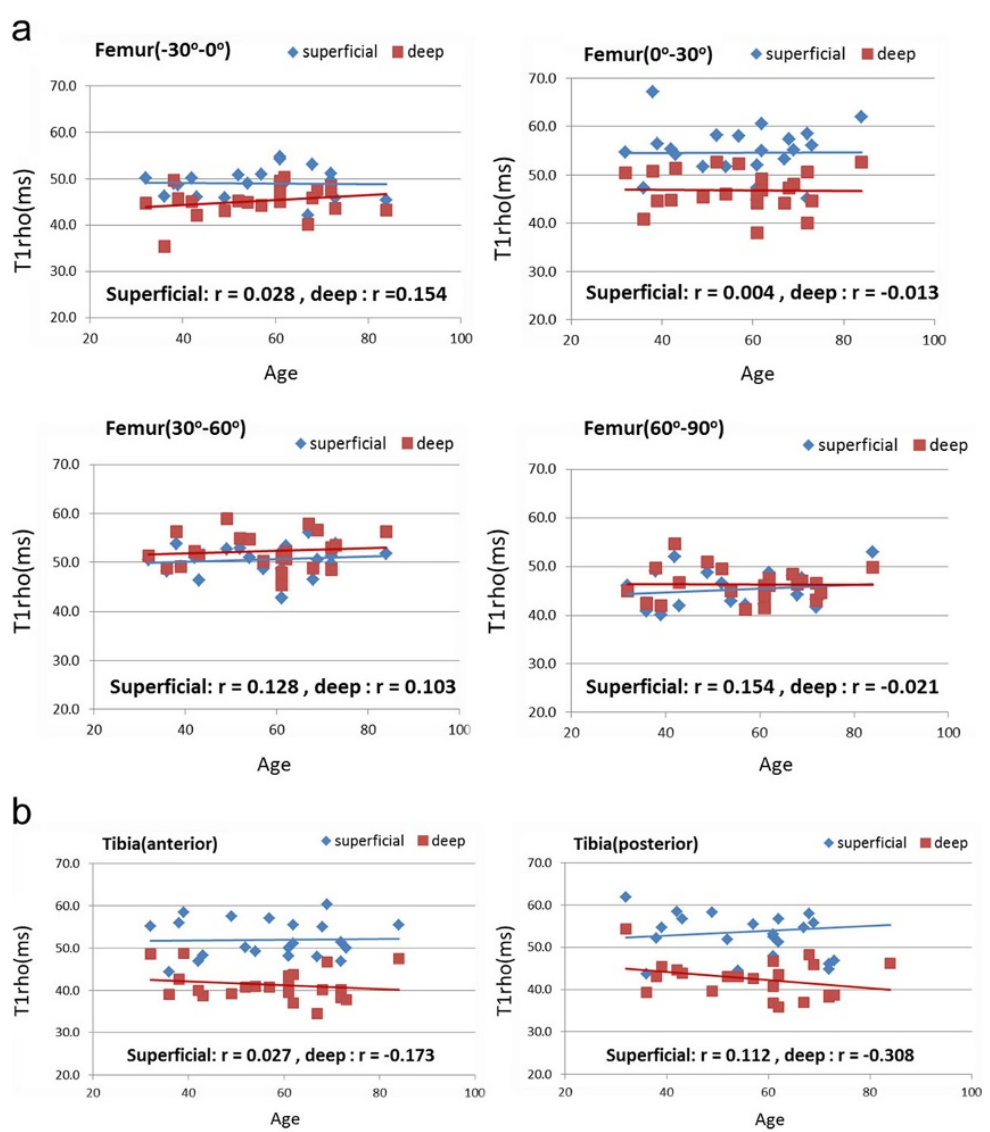

C
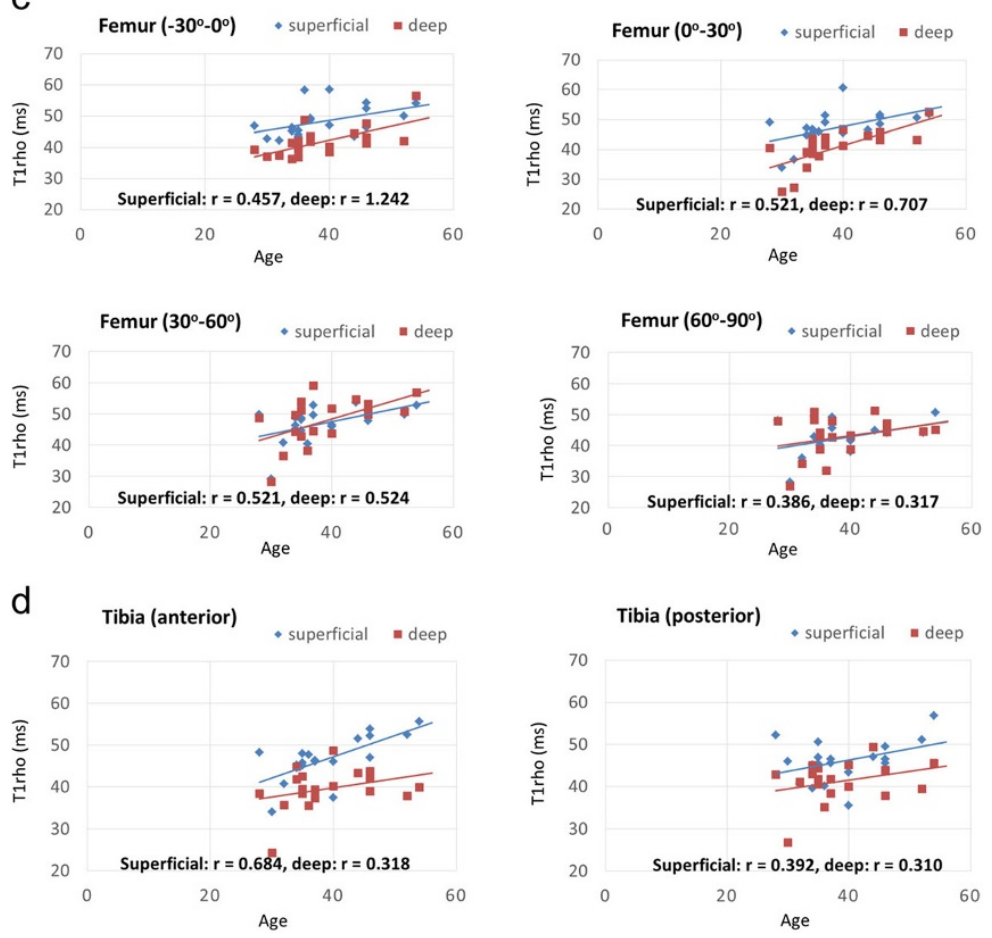

Figure 4 The relationship between age and T1 $\rho$ values of different cartilage regions. $\mathbf{a}$. Femoral condyle of the meniscal tear group. $\mathbf{b}$. Tibial plateau of the meniscal tear group. c. Femoral condyle of the control group. d. Tibial plateau of the control group. 
cartilage disorders in OA knees [21]. T1p-weighted MRI methods have been proposed recently as an attractive potential biomarker to evaluate biochemical changes in the cartilage matrix non-invasively $[10,11]$. It has been reported that $\mathrm{T} 1 \rho$ relaxation time is sensitive to early biochemical changes in cartilage, especially the PG content $[12,15]$. In the early stages of OA, PG depletion occurs prior to the minor structural changes of collagen (denatured collagen) [15]. T1 $\rho$ MRI mapping is a useful imaging method to detect this PG depletion, and minor changes of denatured collagen may also contribute to the T1 $\rho$ signal. Takayama et al. reported that $\mathrm{T} 1 \rho$ mapping is superior to T2 mapping for the assessment of denatured articular cartilage in the early stages of OA, with a capability to assess the severity of cartilage degeneration with good accuracy [22]. This study utilized T1 $\rho$ MRI mapping to detect early cartilage damage in patients with degenerative meniscal tears and healthy subjects.

Some investigators have compared T1 $\rho$ values of subregions and the whole area of the femoro-tibial cartilage and menisci in patients with OA [23], whereas others investigated the relationship between $\mathrm{T} 2$ relaxation values within the superficial zone of the articular cartilage following different types of meniscal degeneration/tear [24]. However, most of these previous studies assessed a limited or region-specific area of the entire joint, such as in one slice of the mid-sagittal plane of the medial and lateral femoral condyles. Although it is known that the severity of degeneration is heterogeneous and focal within the OA joint, there is still a lack of information about the regions of the weight-bearing area in the femoral condyles and the tibia plateaus that are likely to be affected by the early degenerative changes associated with a meniscal tear. Furthermore, superficial and deep layer-specific assessment of the articular cartilage might be more sensitive in terms of detecting early changes in the cartilage macromolecular structures. In the present study, femoral articular cartilage in the meniscal tear group had significantly greater $\mathrm{T} 1 \rho$ values at the distal area, both superficially $\left(-30^{\circ}-0^{\circ}, 0^{\circ}-30^{\circ}\right.$ and $30^{\circ}-60^{\circ}$ areas) and in the deep regions $\left(-30^{\circ}-0^{\circ}\right.$ and $0^{\circ}-30^{\circ}$ areas). In tibial articular cartilage, significantly higher $\mathrm{T} 1 \rho$ values were found superficially in both anterior and posterior areas in the meniscal tear group than in the control group. In both femoral and tibial articular cartilage, no significant differences in T1 $\rho$ values between the two subgroups of OA severity in the meniscal tear group were observed. This finding suggests that degenerative meniscal tears are associated with occult cartilage damage in the very early stages of OA rather than radiographic OA severity, and supports the previous studies that have reported the direct interaction between meniscal and cartilaginous abnormality $[1,8,23,24]$. Hence, occult cartilage damage exists in patients with a symptomatic meniscus tear even though there is no evidence of radiographic OA. The meniscus plays an important role in maintaining the integrity of the articular cartilage by reducing the contact impact forces between the articular surfaces. Damage to the meniscus increases the contact peak stress on the femoral and tibial articular cartilage surfaces and alters the biomechanical loading of the joint, putting the patient at increased risk of cartilage degeneration $[18,25]$.

Previous studies using T1 $\rho$ or T2 MRI mapping have demonstrated a relationship between meniscal and cartilage morphology as well as the cartilage biochemical composition [23,24]. Wang et al. demonstrated that regional damage of both femorotibial cartilage and menisci was associated with the severity of OA using T1p MRI mapping in subjects with various stages of OA [23]. They reported that the T1 $\rho$ values of femoral anterior cartilage sub-region and the medial posterior sub-region of the meniscus are both higher in moderate-severe OA than those in doubtful-mild OA. However, it was unclear whether symptomatic meniscal tear was associated with occult cartilage damage in patients without OA. Kai et al. established an association between meniscal signalcomplex tears and increased $\mathrm{T} 2$ values in tibial articular cartilage [24]. They reported that T2 values of femoral condyle cartilage was not associated with meniscal tear. The differences between $\mathrm{T} 1 \rho$ and $\mathrm{T} 2$ in the sensitivity and diagnostic power for the detection of early macromolecular changes in cartilage might have caused the discrepancy between their results and ours. Furthermore, research into the investigation of the heterogeneity of cartilage $\mathrm{T} 1 \rho$ values in relation to joint morphology is still new. This study suggests that a heterogeneous distribution of cartilage denaturisation associated with meniscal degenerative tears is a precursor of future OA progression.

Additionally, the facts obtained in this study suggest a potential risk for progression of cartilage damage after a meniscal surgery for these patients despite no radiographic OA being observed. Clinical application of this MRI technique as a routine evaluation for meniscal tear could be a help for surgeons. A longitudinal study should be able to reveal whether preoperative evaluation of cartilage macromolecular quality using this MRI technique can predict postoperative progression of cartilage damage.

\section{Conclusions}

This study suggested that degeneration of the cartilage matrix had already occurred in patients with degenerative meniscal tears although morphological changes of the articular cartilage had not been detected. This change appeared in the superficial zone of the articular 
cartilage at the point where femoral and tibial cartilage contact each other during slight flexion. Our results suggest that this area is the origin of early biochemical changes leading to $\mathrm{OA}$ progression associated with degenerative meniscal tears.

\section{Abbreviations}

OA: Osteoarthritis; MRI: Magnetic resonance imaging; PG: Proteoglycan; KL: Kellgren-Lawrence; ICC: Interclass correlation coefficient; ROI: Region of interests.

\section{Competing interests}

The authors declare that they have no competing interests.

\section{Authors' contributions}

HM carried out the image analysis of MRI, participated in the data management and drafted the manuscript. KO (Ken Okazaki) conceived of the study, and participated in its design and coordination and helped to draft the manuscript. YT carried out the reconstruction of T1 $\rho$ Mapping MRI and helped to draft the manuscript. KO (Kanji Osaki) participated in the measure of T1 $\rho$ values and the statistical analysis and helped to draft the manuscript. YM and $\mathrm{HH}$ participated in the clinical examination including MRI and radiography and helped to draft the manuscript. YI involved in revising the manuscript critically for important intellectual content. All authors read and approved the final manuscript.

\section{Acknowledgements}

This study was partly supported by the Grant of Japan Sports Medicine Foundation, 2013, and the Health and Labour Sciences Research Grants for Research on Regenerative Medicine for Clinical Application from the Ministry of Health, Labour and Welfare (grant number: 26220101). The English in this manuscript was edited by Crimson Interactive Pvt. Ltd.

\section{Author details}

${ }^{1}$ Department of Orthopedic Surgery, Graduate School of Medical Sciences, Kyushu University, 3-1-1 Maidashi, Higashi-ku, Fukuoka 812-0054, Japan. ${ }^{2}$ Department of Clinical Radiology, Graduate School of Medical Sciences, Kyushu University, 3-1-1 Maidashi, Higashi-ku, Fukuoka 812-0054, Japan.

Received: 22 August 2014 Accepted: 30 January 2015

Published online: 10 February 2015

\section{References}

1. Christoforakis J, Pradhan R, Sanchez-Ballester J, Hunt N, Strachan RK. Is there an association between articular cartilage changes and degenerative meniscus tears? Arthroscopy. 2005;21:1366-9.

2. Walker PS, Erkman MJ. The role of the menisci in force transmission across the knee. Clin Orthop Relat Res. 1975;109:184-92.

3. Radin EL, de Lamotte F, Maquet P. Role of the menisci in the distribution of stress in the knee. Clin Orthop Relat Res. 1984;185:290-4.

4. Menezes NM, Gray ML, Hartke JR, Burstein D. T2 and T1rho MRI in articular cartilage systems. Magn Reson Med. 2004;51:503-9.

5. Englund M, Guermazi A, Gale D, Hunter DJ, Aliabadi P, Clancy M, et al. Incidental meniscal findings on knee MRI in middle-aged and elderly persons. N Engl J Med. 2008;359:1108-15.

6. Bhattacharyya T, Gale D, Dewire P, Totterman S, Gale ME, McLaughlin S, et al. The clinical importance of meniscal tears demonstrated by magnetic resonance imaging in osteoarthritis of the knee. J Bone Joint Surg Am. 2003;85:4-9.

7. Ding C, Martel-Pelletier J, Pelletier JP, Abram F, Raynauld JP, Cicuttini F, et al. Meniscal tear as an osteoarthritis risk factor in a largely non-osteoarthritic cohort: a cross-sectional study. J Rheumatol. 2007;34:776-84.

8. Hunter DJ, Zhang YQ, Niu JB, Tu X, Amin S, Clancy M, et al. The association of meniscal pathologic changes with cartilage loss in symptomatic knee osteoarthritis. Arthritis Rheum. 2006;54:795-801.

9. Armstrong CG, Mow VC. Variations in the intrinsic mechanical properties of human articular cartilage with age, degeneration, and water content. J Bone Joint Surg Am. 1982;64:88-94.

10. Burstein D, Bashir A, Gray ML. MRI techniques in early stages of cartilage disease. Invest Radiol. 2000;35:622-39.
11. Li X, Benjamin Ma C, Link TM, Castillo DD, Blumenkrantz G, Lozano J, et al. In vivo T1rho and T2 mapping of articular cartilage in osteoarthritis of the knee using 3 T MRI. Osteoarthr Cart. 2007;15:789-97.

12. Akella SV, Regatte RR, Gougoutas AJ, Borthakur A, Shapiro EM, Kneeland JB, et al. Proteoglycan-induced changes in T1rho-relaxation of articular cartilage at 4 T. Magn Reson Med. 2001;46:419-23.

13. Pakin SK, Xu J, Schweitzer ME, Regatte RR. Rapid 3D-T1rho mapping of the knee joint at 3.0 T with parallel imaging. Magn Reson Med. 2006;56:563-71.

14. Blumenkrantz G, Majumdar S. Quantitative magnetic resonance imaging of articular cartilage in osteoarthritis. Eur Cell Mater. 2007;13:76-86.

15. Tsushima H, Okazaki K, Takayama Y, Hatakenaka M, Honda H, Izawa T, et al. Evaluation of cartilage degradation in arthritis using T1 $\rho$ magnetic resonance imaging mapping. Rheumatol Int. 2012;32:2867-75.

16. Peterfy CG, Guermazi A, Zaim S, Tirman PF, Miaux Y, White D, et al. Whole-organ magnetic resonance imaging score (WORMS) of the knee in osteoarthritis. Osteoarthr Cart. 2004;12:177-90.

17. Scarvell JM, Smith PN, Refshauge KM, Galloway HR, Woods KR. Evaluation of a method to map tibiofemoral contact points in the normal knee using MRI. J Orthop Res. 2004;22:788-93.

18. Yao J, Lancianese SL, Hovinga KR, Lee J, Lerner AL. Magnetic resonance image analysis of meniscal translation and tibiomenisco-femoral contact in deep knee flexion. J Orthop Res. 2008;26:673-84.

19. Mosher TJ, Smith H, Dardzinski BJ, Schmithorst VJ, Smith MB. MR imaging and T2 mapping of femoral cartilage: in vivo determination of the magic angle effect. Am J Roentgenol. 2001;177:665-9.

20. Buck FM, Bae WC, Diaz E, Du J, Statum S, Han ET, et al. Comparison of T1rho measurements in agarose phantoms and human patellar cartilage using 2D multislice spiral and 3D magnetization prepared partitioned k-space spoiled gradient-echo snapshot techniques at 3 T. Am J Roentgenol. 2011;196:W179-179.

21. Bassiouni HM, El-Deeb M, Kenawy N, Abdul-Azim E, Khairy M. Phonoarthrography, musculoskeletal ultrasonography, and biochemical biomarkers for the evaluation of knee cartilage in osteoarthritis. Mod Rheumatol. 2011;21:500-8.

22. Takayama Y, Hatakenaka M, Yoshiura T, Okazaki K, Yoshimura T, Yonezawa $M$, et al. T1 $\rho$ is superior to T2 mapping for the evaluation of articular cartilage denaturalization with osteoarthritis: Radiological-pathological correlation after total knee arthroplasty. Eur J Radiol. 2013;82:192-8.

23. Wang L, Chang G, Xu J, Vieira RL, Krasnokutsky S, Abramson S, et al. T1rho MRI of menisci and cartilage in patients with osteoarthritis at 3 T. Eur J Radiol. 2012:81:2329-36.

24. Kai B, Mann SA, King C, Forster BB. Integrity of articular cartilage on T2 mapping associated with meniscal signal change. Eur J Radiol. 2011;79:421-7.

25. Maquet PG, Van de Berg AJ, Simonet JC. Femorotibial weight-bearing areas. Experimental determination. J Bone Joint Surg Am. 1975;57:766-71.

\section{Submit your next manuscript to BioMed Central and take full advantage of:}

- Convenient online submission

- Thorough peer review

- No space constraints or color figure charges

- Immediate publication on acceptance

- Inclusion in PubMed, CAS, Scopus and Google Scholar

- Research which is freely available for redistribution 